\title{
Simultaneous acousto-optic diffractions by two lobes of a shaped phased-array transducer
}

\author{
Illienko M. O., Pilgun Yu. V. and Smirnov Ye. M. \\ Faculty of Radiophysics, Electronics and Computer Systems, Taras Shevchenko \\ National University of Kyiv, 64/13 Volodymyrska Street, 01601 Kyiv, Ukraine \\ maksym.illienko@knu.ua
}

Received: 26.04 .2021

\begin{abstract}
We consider simultaneous diffractions of light by two lobes of sound radiation pattern in an acousto-optic modulator which is built on $\mathrm{SiO}_{2}$ single crystal and has two-element phased-array transducer with the fixed phase shift $\pi$. Implementation of this diffraction type greatly improves power efficiency of the device. Transducer shaping is used to steer the lobes and satisfy the Bragg's conditions for simultaneous acousto-optic diffractions. The conditions of the diffraction are defined and a mathematical model for calculating diffraction efficiency is suggested. It is shown that a parallel diffraction regime is almost three times more energy-efficient than a serial regime. Several configurations of transducer elements' shape are analyzed. In particular, changes in the dimensions of diamond-shaped elements are shown to allow adjusting the operating frequency within $23 \%$. An experimental sample of the device is built.
\end{abstract}

Keywords: acousto-optic diffraction, phased-array transducer, transducer shaping, lobes of radiation pattern, simultaneous diffraction, diffraction efficiency.

UDC: $535.42,534.522 .1$

\section{Introduction}

Use of phased-array transducers is one of the most common ways to improve characteristics of acousto-optic (AO) devices [1-5]. Phase manipulation of each transducer cell allows one to modify acoustic radiation pattern. Therefore, reduction of side-lobe intensities and beam steering can be achieved, thus resulting in expanded dynamic range and bandwidth of AO devices [1-5]. Although the best results can be reached when the phase and the amplitude of cells are controlled electronically [5], simply connecting piezoelectric cells in a series with the opposite polarities, which results in the phase shift $\pi$, also increases the bandwidth and the dynamic range [2]. To implement this phase shift, a metalized binding layer between a piezoelectric plate and a crystal can be used to connect two parts of a transducer in the opposite direction. The electrodes delivering a signal are sputtered on a top of the piezoelectric plate, thus realizing two-element phased-array transducer with a fixed phase shift $\pi$.

In this simplest configuration, a bottom electrode does not deliver electrical power and all the power is delivered through a top electrode, which allows one to satisfy higher power requirements for quartz-based AO modulators. A significant disadvantage of such transducers is a presence of two acoustic-beam lobes. However, only one of these lobes is normally used in AO interactions so that the energy of the other lobe is wasted.

In the present work, we demonstrate a possibility for simultaneous light diffractions by the both acoustic lobes in a quartz AO modulator with a two-element transducer. This diffraction occurs under a certain condition that relies upon a link among the sound frequency, the light 
wavelength, the transducer size and its shape. As the sound frequency and the light wavelength are usually fixed, the simultaneous diffraction regime can be achieved by adjusting the size or the shape of transducer. Although here we consider only a two-element transducer, our methods can be extended to the case of any number of those elements.

\section{Condition of simultaneous diffractions by both side lobes}

The sound-radiation pattern of two-element transducer with the phase shift $\pi$ consists of two directional lobes (see Fig. 1a). The parameters of the incident light and the acoustic frequency can be chosen in order to satisfy the Bragg's condition for the both lobes (see Fig. 1b, c). In this case, simultaneous diffractions by the both directional lobes occur. One can see from Fig. 1 that the two regimes of simultaneous diffraction are possible: parallel (Fig. 1b) and serial (Fig. 1c) ones. In a parallel regime, the incident light beam specified by the wavevector $\boldsymbol{k}_{\boldsymbol{i}}$ is diffracted by the left $\left(\boldsymbol{K}_{L}\right)$ and right $\left(\boldsymbol{K}_{R}\right)$ sound lobes into the $+1^{\text {st }}$ and $-1^{\text {st }}$ orders, respectively. In a serial regime, the incident beam diffracts into the $+1^{\text {st }}$ order and then by the other lobe into the $+2^{\text {nd }}$ order. Naturally, the lengths of the vectors $\boldsymbol{K}_{L}$ and $\boldsymbol{K}_{\boldsymbol{R}}$ and the angle $\psi$ between them depend on the sound frequency. Besides, $\psi$ is also affected by the transducer size and shape. Hereby, there should be some condition, like the Bragg's one, which relates the parameters of light, sound and transducer to match the vector diagrams in Fig. 1. Let us find this condition.

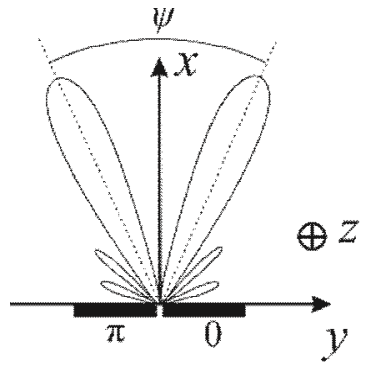

a)

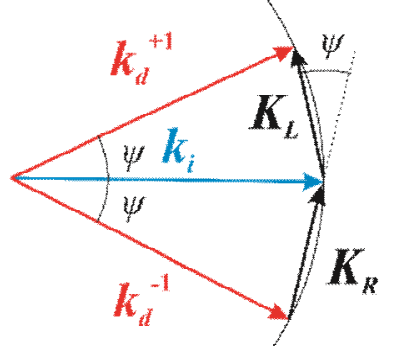

b)

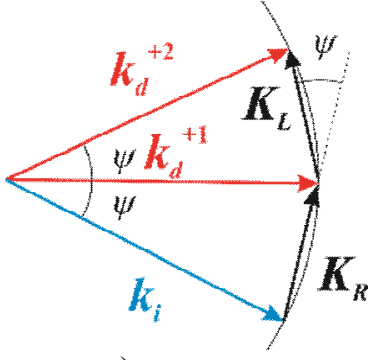

c)

Fig. 1. Simultaneous light diffraction by two acoustic lobes: sound radiation pattern (a) and vector diagrams for parallel (b) and serial (c) diffractions. The angle $\psi$ between the sound lobes is twice as large as the Bragg's angle $\theta_{B}$.

To start, let us remark that the difference between the parallel and serial diffractions consists only in the selection of incident light beam. Therefore, the condition we are going to define should be the same for the both cases. Let the transducer face be placed in the $y z$ plane and the acoustic wave propagate along the $x$ axis. The radiation pattern $I(\varphi, \theta)$ of transducer can be defined by Fourier transform of distribution of the displacement-vector amplitude in the transducer plane (here we suppose the amplitude to be constant and unit within the bounds of transducer):

$$
I(\varphi, \theta)=\left|\frac{1}{S} \int_{S} e^{-j\left(K_{y} y+K_{z} z\right)} d y d z\right|^{2}=\left|\frac{1}{S} \int_{S} e^{-j K(\sin \theta \sin \varphi y+\cos \theta z)} d y d z\right|^{2},
$$

where $S$ denotes the transducer area, $K$ the acoustic wavenumber, and $\varphi$ and $\theta$ are respectively the azimuthal and zenithal angles (see Fig. 2). Since the light diffraction takes place in the $x y$ plane, the acoustic radiation pattern should also be examined in this plane. Thus, assuming $\theta=\pi / 2$ and $K_{z}=0$, one can transform the surface integral in Eq. (1) into the interval one (see Fig. 2) 


$$
\begin{aligned}
& I\left(\varphi, \frac{\pi}{2}\right)=I(\varphi)=\left|\frac{1}{S} \int_{S} e^{-j K_{y} y} d y d z\right|^{2}=\left|\frac{1}{S} \int_{a}^{b} e^{-j K_{y} y} d y \int_{v(y)}^{u(y)} d z\right|^{2} \\
& =\left|\frac{1}{S} \int_{a}^{b}(u(y)-v(y)) e^{-j K_{y} y} d y\right|^{2}=\left|\frac{1}{S} \int_{a}^{b}(u(y)-v(y)) e^{-j K \sin \varphi y} d y\right|^{2},
\end{aligned}
$$

where the functions $u(y): y \rightarrow z$ and $v(y): y \rightarrow z$ determine the transducer shapes above and below the $y$ axis, respectively (see Fig. 2).

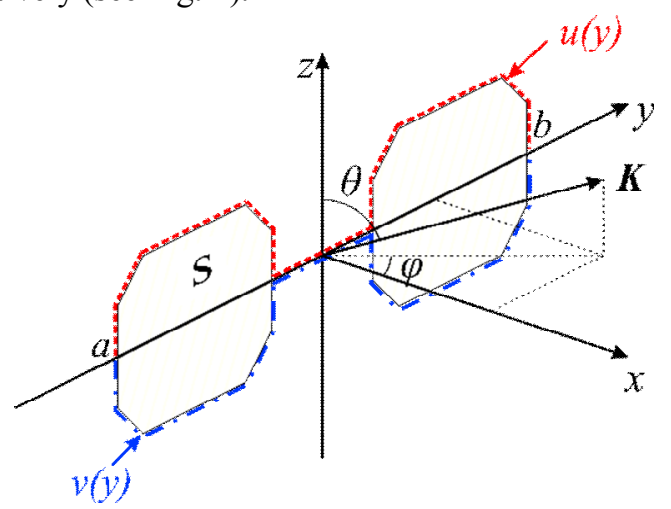

Fig. 2. Two-element transducer geometry. Hatched are elements with the opposite phase and $\boldsymbol{K}$ denotes acoustic wavevector.

Exploring the extrema of Eq. (2), one can obtain the angle $\varphi^{\text {opt }}=\psi / 2$ (see Fig. 1a) and the acoustic wavevector projection $K_{y}^{\text {opt }}$ that correspond to the main sound lobes. Due to symmetry of our problem, the wavevector projections $K_{y}^{\text {opt }}$ for the left and right lobes differ by only their signs. Further on, we consider only the positive lobe. The $K_{y}^{\text {opt }}$ value depends on the size and shape of transducer and remains independent of the acoustic frequency. One can find from Fig. 1 that the Bragg's condition for the both acoustic lobes is as follows:

On the other hand, we have

$$
\sin \frac{\psi}{2}=\sin \varphi^{o p t}=\frac{K}{2 k} \text {. }
$$

$$
\sin \varphi^{o p t}=\frac{K_{y}^{o p t}}{K} .
$$

Combining Eqs. (3) and (4) and introducing the notations $K=2 \pi f / V$ and $k=2 \pi n / \lambda$, we arrive at

$$
f=\sqrt{\frac{n V^{2}}{\lambda \pi} K_{y}^{o p t}},
$$

with $f$ being the acoustic frequency, $V$ the phase velocity of sound, $n$ the refractive index of a material medium, and $\lambda$ the light wavelength. Eq. (5) summarizes the condition for the simultaneous diffraction.

Let the transducer size defined by the limits of integration $a$ and $b$ in Eq. (2) be fixed, which is generally satisfied. Then it would be reasonable to exclude the effect of $a$ and $b$ on the $K_{y}^{o p t}$ parameter. The scaling property of the Fourier transform enables rewriting Eq. (2) as 


$$
I\left(K_{n}\right)=\left|\frac{1}{S l} \int_{0}^{1}(u(a+l y)-v(a+l y)) e^{-j K_{n} y} d y\right|^{2}, \quad l=|a-b| .
$$

Although Eq. (6) contains $a$ and $b$, it is clear that the functions $u(a+l y)$ and $v(a+l y)$ can be redefined as $u^{\prime}\left(y^{\prime}\right)$ and $v^{\prime}\left(y^{\prime}\right)$ for a normalized coordinate $y^{\prime} \in[0 ; 1]$. The appropriate new functions describe the transducer shape independent of its dimension along the $y$ axis. Similarly to Eq. (2), a corresponding $K_{n}^{\text {opt }}$ value, which does not depend on the absolute value of $l$, can be found from Eq. (6). The $K_{n}^{o p t}$ and $K_{y}^{\text {opt }}$ parameters are related as follows:

$$
K_{y}^{o p t}=K_{n}^{o p t} / l \text {. }
$$

One can transform Eq. (5) to obtain

$$
f=\sqrt{\frac{n V^{2}}{\lambda \pi l} K_{n}^{o p t}}
$$

It is worthwhile to note that simultaneous multiplication of the functions $u^{\prime}\left(y^{\prime}\right)$ and $v^{\prime}\left(y^{\prime}\right)$ in Eq. (6) by a positive constant, which corresponds to scaling of transducer shape along the $z$ axis, does not affect the $K_{n}^{\text {opt }}$ value and, therefore, the directions of the main sound lobes. Hence, the $K_{n}^{\text {opt }}$ value is determined by the transducer shape defined up to a constant factor by the functions $u^{\prime}\left(y^{\prime}\right)$ and $v^{\prime}\left(y^{\prime}\right)$. Consequently, the variation of transducer shape can be used to satisfy Eq. (8) provided that the other transducer parameters (e.g., the acoustic frequency and the size) are kept fixed.

\section{Diamond-shaped transducer elements}

Transducer with its elements shaped as a diamonds is commonly used to reduce the level of unwanted acoustic side lobes [6,7]. Apart from acoustic-beam improvement, such a transducer shape is convenient for implementing simultaneous diffractions by the two acoustic lobes. Variation of the $\alpha$ parameter (see Fig. 3) leads to change in the azimuthal angle $\varphi^{o p t}=\psi / 2$. For any predefined acoustic frequency, one can select such $\alpha$ that the angle $\psi$ between the sound
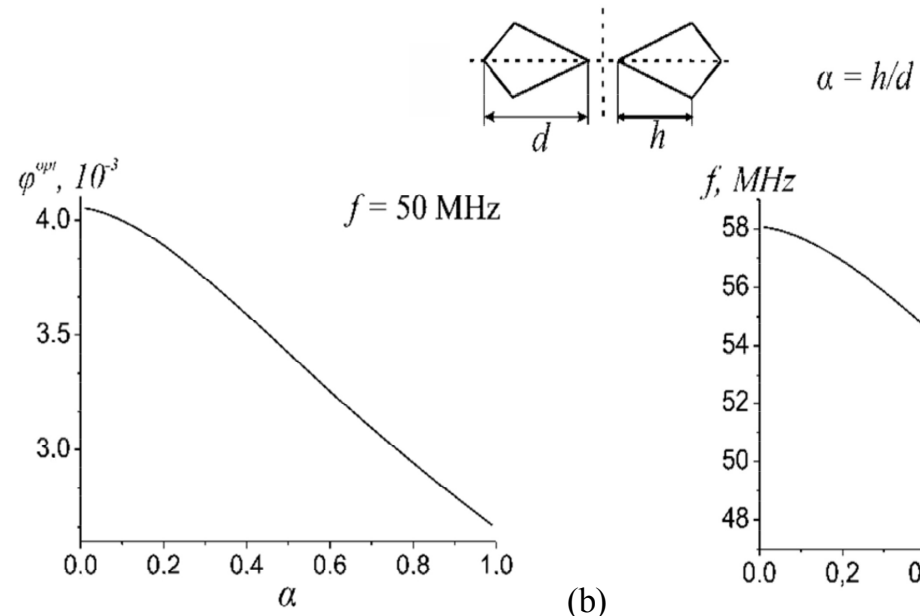

(b)

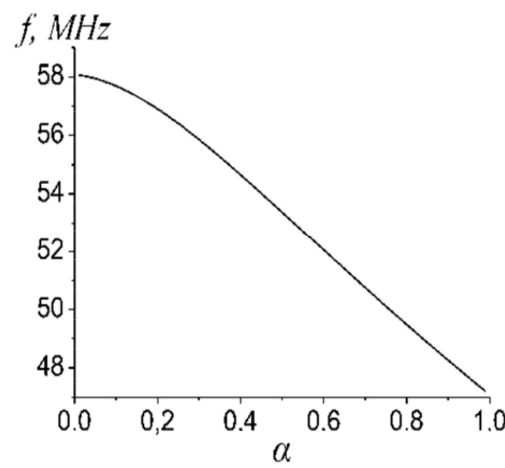

(c)

Fig. 3. Transducer with diamond-shaped elements: (a) dimensions of elements, (b) dependence of beamdirection angle $\varphi^{\text {opt }}$ on dimension ratio $\alpha$ at the acoustic frequency $50 \mathrm{MHz}$, and (c) acoustic-frequency dependence on $\alpha$. Dimension $d$ is equal $14 \mathrm{~mm}$. Light wavelength is equal to $1064 \mathrm{~nm}$. Note: variation of $\alpha$ implies only change in $h$ value and the other transducer dimensions are fixed.

Ukr. J. Phys. Opt. 2021, Volume 22, Issue 3 
lobes would satisfy the Bragg's condition for the both sound lobes. The range of attainable $\varphi^{\text {opt }}$ values at the acoustic frequency $50 \mathrm{MHz}$ is shown in Fig. 3a. Contrariwise, the range of acoustic frequencies for which the simultaneous diffraction is possible can be determined using Eq. (8) and varying the $\alpha$ value from 0 to 1 (see Fig. 3b). From Eq. (6) one can determine the $K_{n}^{\text {opt }}$ values at $\alpha=0$ and $\alpha=1$. They are equal to $\pi$ and $\approx 2.082$ respectively. According to Eq. (8), the ratio of the maximum frequency to the minimum one is $\sqrt{\pi / 2.082} \approx 1.23$. This estimation of attainable frequency variation remains general for any acoustically isotropic medium, being independent of the parameters of light and material medium. Examples of radiation patterns observed in the cases of $\alpha=1 / 2$ and $\alpha=5 / 7$ are shown in Fig. 4 .
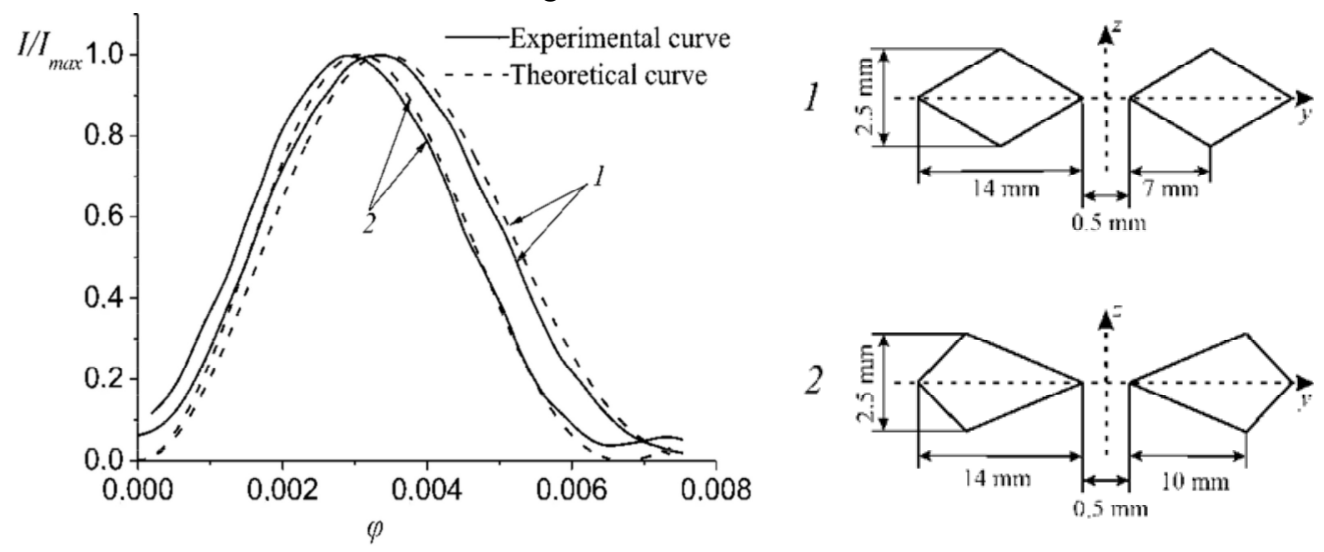

Fig. 4. Radiation patterns obtained for the cases of two differently shaped transducers. Acoustic wave propagates along the $x$ axis in a $\mathrm{SiO}_{2}$ crystal. The acoustic frequency is equal to $50 \mathrm{MHz}$. The difference between the lobe directions 1 and 2 is $5 \times 10^{-4}$ rad. This corresponds to $K_{n}^{\text {opt }}$ change of $18 \%$ in the case 2 , when compared with the case 1.

\section{Diffraction efficiency}

To calculate the diffraction efficiency, a matrix formalism described in Ref. [8] has been used. The concept of this method is that each transducer element forms a separate AO cell, whose output light amplitudes represent input ones for a following cell (see Fig. 5). The relation between the complex amplitudes of the input and output lights in each cell is represented by a matrix. Therefore, the light amplitudes after passing all AO cells can be found by a chain product of the corresponding matrixes.

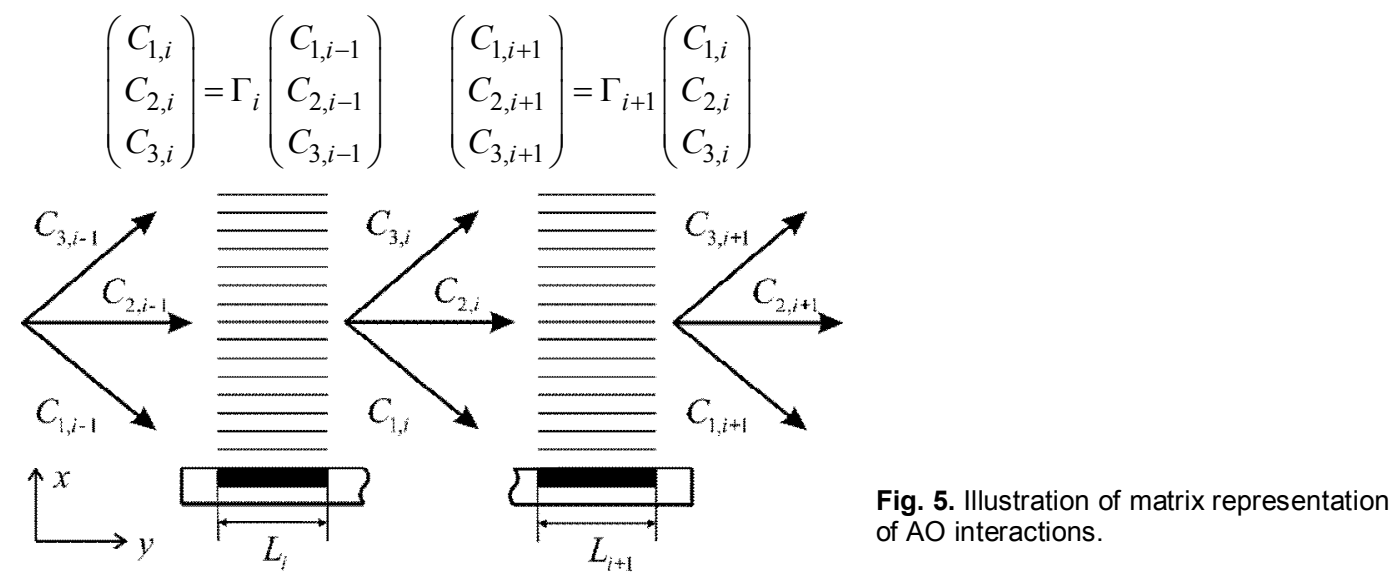




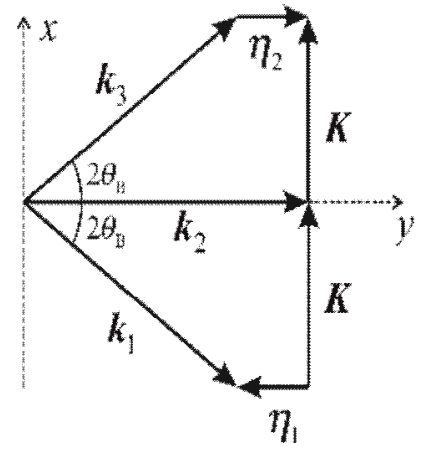

Fig. 6. Vector diagram of $A O$ interaction within a single cell.

Let us consider AO interaction within a single cell. In our case, there are three light beams. Their interaction diagram is illustrated in Fig. 6. We are not obliged to specify either parallel or serial diffractions, since the physical coupling between the light beams is the same in the both cases. According to Ref. [9], this coupling is described as

$$
\frac{d C_{p}}{d y}=\frac{q}{2}\left(C_{p-1} \exp \left[j\left(\eta_{p-1} y+\Phi\right)\right]-C_{p+1} \exp \left[-j\left(\eta_{p} y+\Phi\right)\right]\right) \text {. }
$$

Here $C_{p}$ is the complex amplitude of the $p$-th light beam, $\eta_{p}=k_{p, y}-k_{p+1, y}$ the mismatch parameter, $\Phi$ the phase of sound, $q \sim \sqrt{P_{a} / S}$ the coupling factor, and $P_{a}$ and $S$ are respectively the acoustic power and the area of transducer element. With the reference to Fig. 6, Eq. (9) can be rewritten as

$$
\begin{aligned}
\frac{d C_{1}}{d y} & =-\frac{q^{*}}{2} C_{2} \exp [j \eta y], \\
\frac{d C_{2}}{d y} & =\frac{q}{2} C_{1} \exp [-j \eta y]-\frac{q^{*}}{2} C_{3} \exp [-j \eta y], \\
\frac{d C_{3}}{d y} & =\frac{q}{2} C_{2} \exp [j \eta y] .
\end{aligned}
$$

where the substitution $q \exp [i \Phi] \rightarrow q$ has been made. Using Fig. 6 and assuming $K \ll k$, one can obtain the relationship $\eta=k^{2}-\sqrt{k^{2}-K^{2}} \approx K^{2} / 2 k$. In common with the work [8], we transform Eq. (11) in the way that provides necessary phase relationships at the right edge of the cell and makes the system of Eqs. (10) simpler:

$$
C_{1}=A_{1} \exp [j \eta y], \quad C_{2}=A_{2}, \quad C_{3}=A_{3} \exp [j \eta y] .
$$

Substitution Eq. (11) into Eq. (10) gives the following coupled-mode equations:

$$
\begin{aligned}
& \frac{d A_{1, i}}{d y_{i}}=-j \eta A_{1, i}-\frac{q_{i}^{*}}{2} A_{2, i}, \\
& \frac{d A_{2, i}}{d y_{i}}=\frac{q_{i}}{2} A_{1, i}-\frac{q_{i}^{*}}{2} A_{3, i}, \\
& \frac{d A_{3, i}}{d y_{i}}=-j \eta A_{3, i}+\frac{q_{i}}{2} A_{2, i} .
\end{aligned}
$$


Here the lower subscript $i$ defines the corresponding AO cell and the coordinate $y_{i}$ corresponds to the position within the $i$ th cell with respect to its left edge. Let us remind that the output light of $(i-1)$ th cell is input for the $i$ th cell. Then the boundary conditions for Eq. (12) are as follows:

$$
\begin{aligned}
& A_{1, i}\left(y_{i}=0\right)=A_{1, i-1}\left(y_{i-1}=L_{i-1}\right)=A_{1}^{i-1}, \\
& A_{2, i}\left(y_{i}=0\right)=A_{2, i-1}\left(y_{i-1}=L_{i-1}\right)=A_{2}^{i-1}, \\
& A_{3, i}\left(y_{i}=0\right)=A_{3, i-1}\left(y_{i-1}=L_{i-1}\right)=A_{3}^{i-1} .
\end{aligned}
$$

The solution of Eq. (12) with the boundary conditions Eq. (13) can be put into matrix form, thus relating the output amplitudes to the input ones in all of the diffraction orders:

$$
\begin{aligned}
& \left(\begin{array}{l}
A_{1}^{i} \\
A_{2}^{i} \\
A_{3}^{i}
\end{array}\right)=\Gamma_{i}\left(\begin{array}{c}
A_{1}^{i-1} \\
A_{2}^{i-1} \\
A_{3}^{i-1}
\end{array}\right),
\end{aligned}
$$

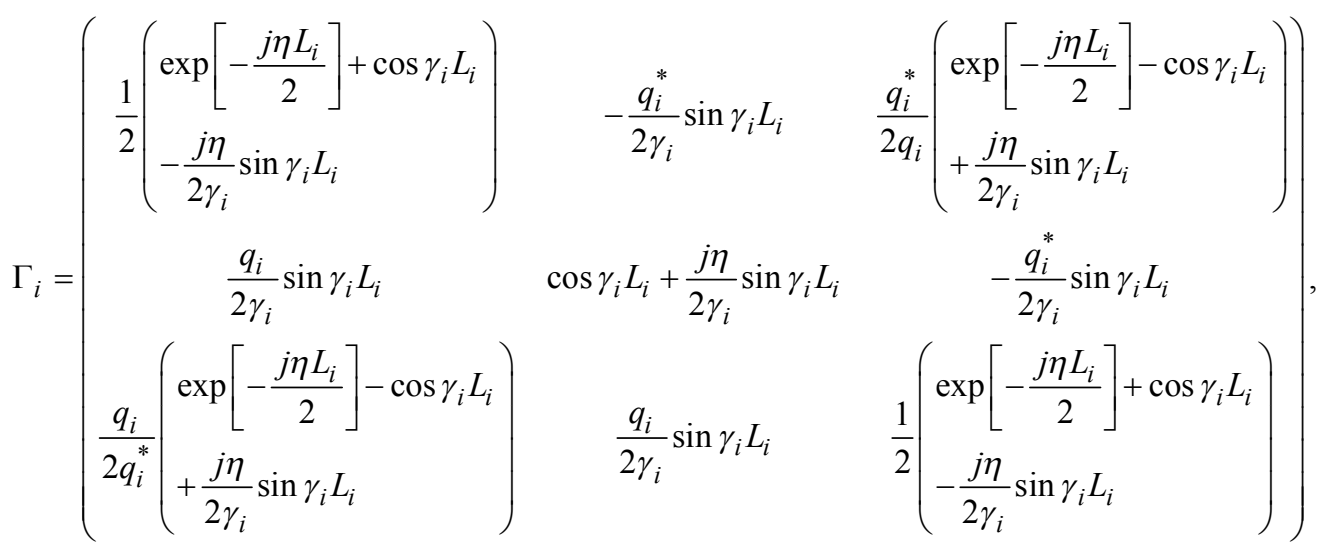

$$
\begin{aligned}
& \gamma_{i}=\frac{\sqrt{\eta^{2}+2\left|q_{i}\right|^{2}}}{2} \text {. }
\end{aligned}
$$

Defining the matrices $\Gamma_{i}$ for all the AO cells, one can calculate the final output-light amplitudes in the following way:

$$
\left(\begin{array}{c}
A_{1}^{\text {out }} \\
A_{2}^{\text {out }} \\
A_{3}^{\text {out }}
\end{array}\right)=\Gamma_{n}\left(q_{n}, L_{n}\right) \Gamma_{n-1}\left(q_{n-1}, L_{n-1}\right) \ldots \Gamma_{1}\left(q_{1}, L_{1}\right)\left(\begin{array}{c}
A_{1}^{\text {in }} \\
A_{2}^{\text {in }} \\
A_{3}^{\text {in }}
\end{array}\right) .
$$

Here the amplitudes $A_{i}^{\text {in }}$ describe the incident light and specify the diffraction regime. When $A_{2}^{i n}=1$ and $A_{1,3}^{i n}=0$, the parallel diffraction occurs. On the contrary, the diffraction becomes serial whenever we have the relations $A_{1}^{i n}=1, A_{2,3}^{i n}=0$ or $A_{3}^{i n}=1, A_{1,2}^{i n}=0$. Note that the zeroorder diffraction corresponds to the subscript $i$ for which we have $A_{i}^{i n}=1$.

Although in our case the transducer consists only of two elements, Eq. (15) may contain more than two matrices $\Gamma_{i}$. The additional matrices appear when taking into account the distance between transducer elements or the shape. The zones with no sound also can be described by the matrix $\Gamma_{i}$ assuming $q_{i}=0$. 
Eq. (15) represents a solution of a one-dimensional diffraction problem and does not involve any transducer shape that differs from rectangular. Non-rectangular transducers can be considered as those in which the length of the AO interaction changes along the direction perpendicular to the diffraction plane [10]. Then the transducer can be divided into thin longitudinal strips (see Fig. 7).

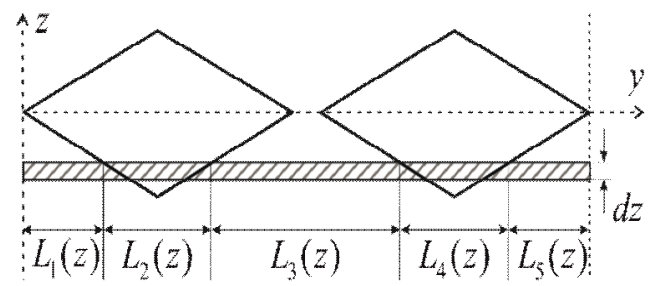

Fig. 7. Dividing laser beam into thin strips. Laser beam propagates along $y$ axis.

For every strip, the partial light intensity is calculated with Eq. (15). The total light intensity is a sum of all the partial components:

$$
\left(\begin{array}{c}
I_{1}^{\text {out }} \\
I_{2}^{\text {out }} \\
I_{3}^{\text {out }}
\end{array}\right)=\frac{1}{2 r} \int_{-r}^{r} \mid \Gamma_{n}\left(q_{n}, L_{n}(z)\right) \Gamma_{n-1}\left(q_{n-1}, L_{n-1}(z)\right) \ldots \Gamma_{1}\left(q_{1}, L_{1}(z)\right)\left(\left.\begin{array}{c}
A_{1}^{\text {in }} \\
A_{2}^{\text {in }} \\
A_{3}^{\text {in }}
\end{array}\right|^{2} d z,\right.
$$

where $r$ is the light-beam radius.

Now let us consider the parallel diffraction regime. The transducer contains two rectangular elements placed next to one another, with the length of each element being equal to $L$. As the phase shift between the elements is $\pi$, the coupling factors are $q_{1}=q$ and $q_{2}=-q$. Using the corresponding initial amplitudes in Eq. (15), one can obtain the intensity of the zero-order beam:

$$
\begin{aligned}
& I_{\text {zero }}=\left|A_{2}^{\text {out }}\right|^{2}=\left|\frac{2 q^{2}+\eta^{2} \cos 2 \gamma L}{4 \gamma^{2}}+j \frac{\eta \sin 2 \gamma L}{2 \gamma}\right|^{2} \\
& =1-\frac{q^{2} \eta^{2} L^{4}}{2}\left[\sin ^{4}\left(L \sqrt{2 q^{2}+\eta^{2}} / 2\right) / \frac{L^{4}{\sqrt{2 q^{2}+\eta^{2}}}^{4}}{2^{4}}\right] \\
& =\left|\begin{array}{l}
2 q^{2} L^{2}=\xi \\
\eta^{2} L^{2}=\zeta
\end{array}\right|=1-\frac{\xi \zeta}{4} \operatorname{sinc}^{4}(\sqrt{\xi+\zeta} / 2) .
\end{aligned}
$$

When we have $\xi=\zeta=\pi^{2} / 2$, Eq. (17) has a null corresponding to a maximal diffraction efficiency. The optimal values of the mismatch parameter $\eta$ and the coupling factor $q$ are then given by

$$
\begin{aligned}
& \eta L=\pi / \sqrt{2} \Rightarrow \eta=\pi / \sqrt{2} L, \\
& q L=\pi / 2 \Rightarrow q=\pi / 2 L .
\end{aligned}
$$

The optimal value $\eta L$ is assumed to be the same for the both parallel and serial regimes. Fig. 8 demonstrates a dependence of zero-order intensity on $q L$ at the $\eta L$ value defined by Eq. (18). It is evident that the optimal acoustic power is almost three times larger in the serial regime when compared to the parallel one. Thus, the parallel regime proves to be more efficient. Below we will consider only this case. 


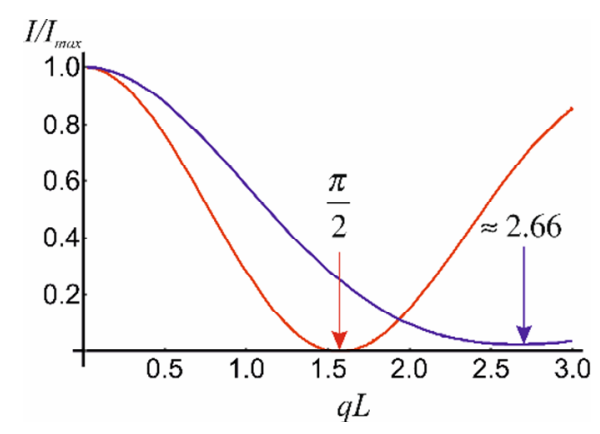

Fig. 8. Output intensity of a zero-order light depending on $q L$ value. Red and blue lines correspond to parallel and serial regimes, respectively. The ratio of positions of 'minimum points' is equal to 1.69 , which corresponds to the power ratio 2.87 .

\section{Acoustic-field distribution}

The diffraction efficiency has been calculated in Section 4, using the most common model of a 'sound column'. It implies a plane acoustic wave with a uniform amplitude bounded by a transducer aperture. Such a model is close to considering AO interaction in the near field in the sense that the acoustic wavefront remains planar. However, it accounts for no inhomogeneity of the acoustic amplitude, which is always present in the near-field region, thus resulting in overestimated diffraction efficiency.

To study the effect of acoustic-amplitude variations, we have examined the acoustic-field distributions for four different transducer configurations (see Fig. 9). Fig. 10 depicts the results of numerical calculations for some 3D amplitude distributions of acoustic beam in $\mathrm{SiO}_{2}$ crystals. A transducer is placed in the $y z$ plane and radiates a $50 \mathrm{MHz}$ longitudinal acoustic wave along the $x$ axis.

Transducer configurations
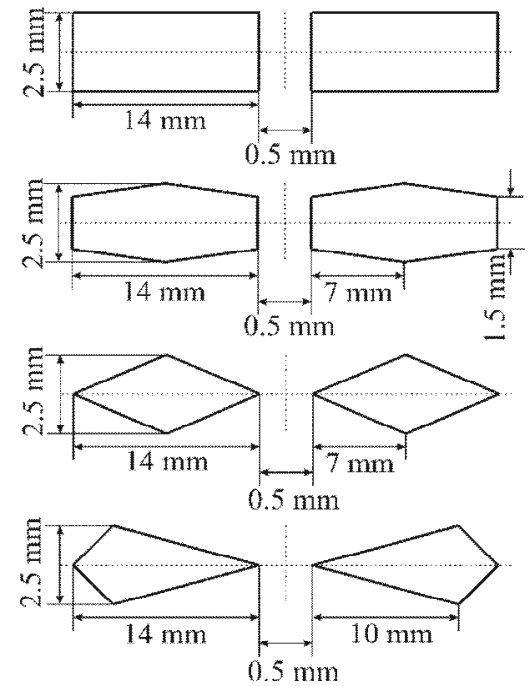

Fig. 9. Transducer configurations for which 3D sound-field distribution is computed.

Three configurations of transducers have been realized in the experiment where twodimensional projections of some acoustic field are experimentally visualized using the AO technique [11] (see insets in Fig. 10). The acoustic field calculated for the fourth configuration with a rectangular transducer is provided for comparison. 


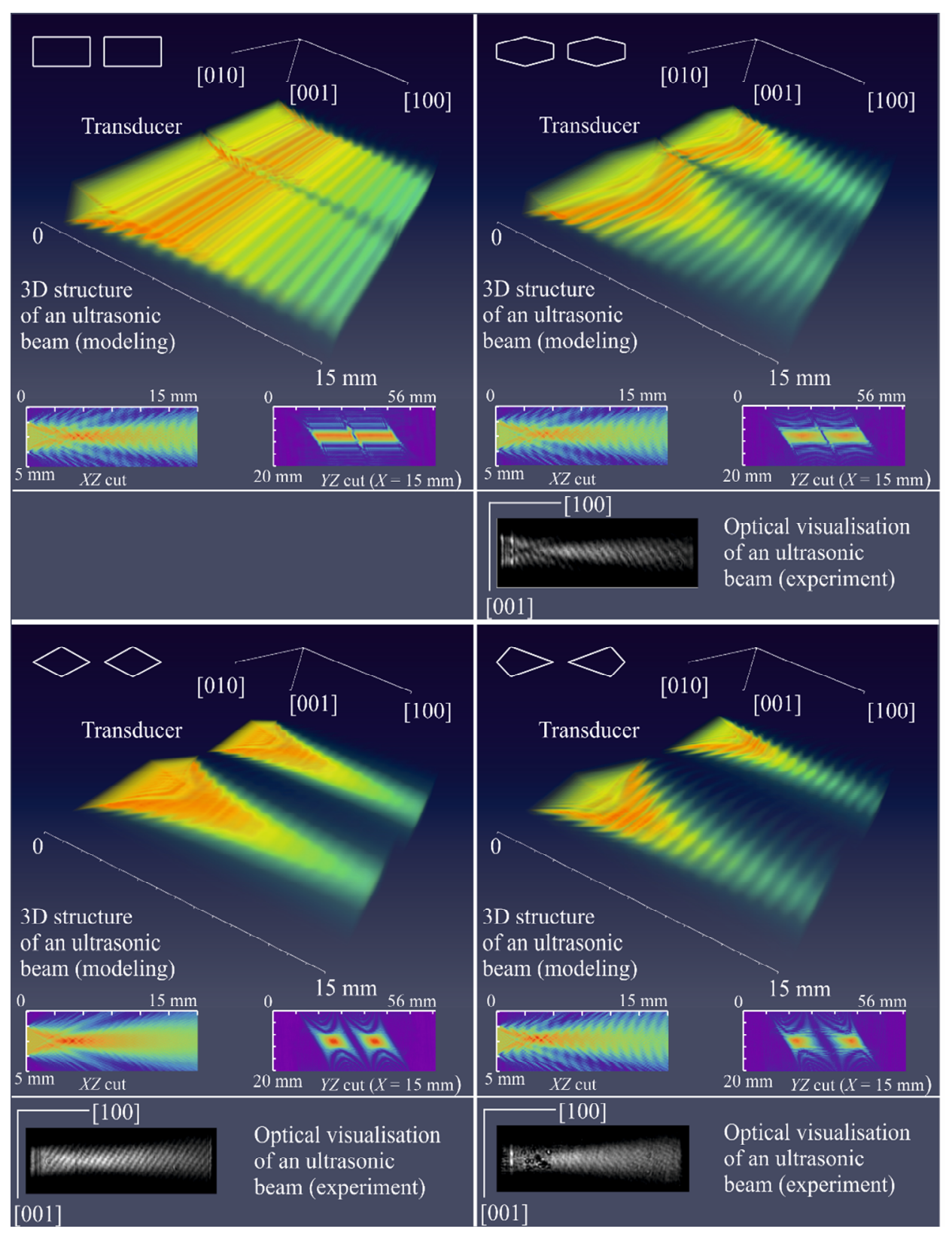

Fig. 10. Amplitude distributions of longitudinal ultrasonic wave in $\mathrm{SiO}_{2}$ crystals. Acoustic frequency is $50 \mathrm{MHz}$. Color palette and transparency settings for 3D images are arbitrarily adjusted for better visualization of the field. Grayscale inserts show experimentally visualized sound fields.

The acoustic-field calculations have been performed using a planar-wave decomposition method that takes acoustic anisotropy into account [12-14]. This method involves Fourier decomposition of the acoustic-displacement field within the radiation (i.e., transducer) plane in order to represent it as a sum of plane waves. The acoustic displacement at any point of half-space can be found by summing up all of the plane waves with the corresponding phase incursions.

It is evident from Fig. 10 that the acoustic beams radiated by neighbouring transducer elements do not intersect. Furthermore, the acoustic beam in the $x y$ cut (i.e., the plane of AO interaction) is almost non-divergent because of high anisotropy of the crystal. The Fresnel region 
where the acoustic wave has almost plane wavefront stretches over approximately $3 \mathrm{~mm}$ from the transducer plane. Away from these $3 \mathrm{~mm}$, the acoustic field forms the main divergent lobe wherein the divergence is mostly present only in the $x z$ plane. The distribution of the displacement amplitude in the beam cross-section is strongly non-uniform due to diffraction and crystal anisotropy. Also, it is skewed along the $y$ axis. The non-uniformity and skewness are not taken into consideration in the model of 'sound column', which is a significant drawback of this model.

Additionally, there is a pronounced modulation of sound amplitude along the $x$ axis. The origin of this modulation is not clear for us, and the same refers to its absence in the case of symmetric diamond-like shaping. Most probably, the effect is caused by destructive interference of partial acoustic waves in a strongly anisotropic medium. The modulation period (approximately $0.9 \mathrm{~mm}$ ) is the same for all the AO-cell configurations considered by us, excluding the case of symmetric diamond shaping. A modulation seen in the experimental patterns is caused by a different effect: this is interference of light transmitted and reflected off the side crystal faces. This optical interference might prevent us from detecting the sound-amplitude modulation obtained in the calculations.

\section{Comparison of experimental and theoretical results}

Fig. 11 demonstrates dependences of the calculated and experimentally measured diffraction efficiencies on the acoustic power, as obtained in the case of parallel diffraction at the light wavelength $632.8 \mathrm{~nm}$ with the light polarization oriented along the $z$ axis. The acoustic frequency is selected according to the transducer shape.
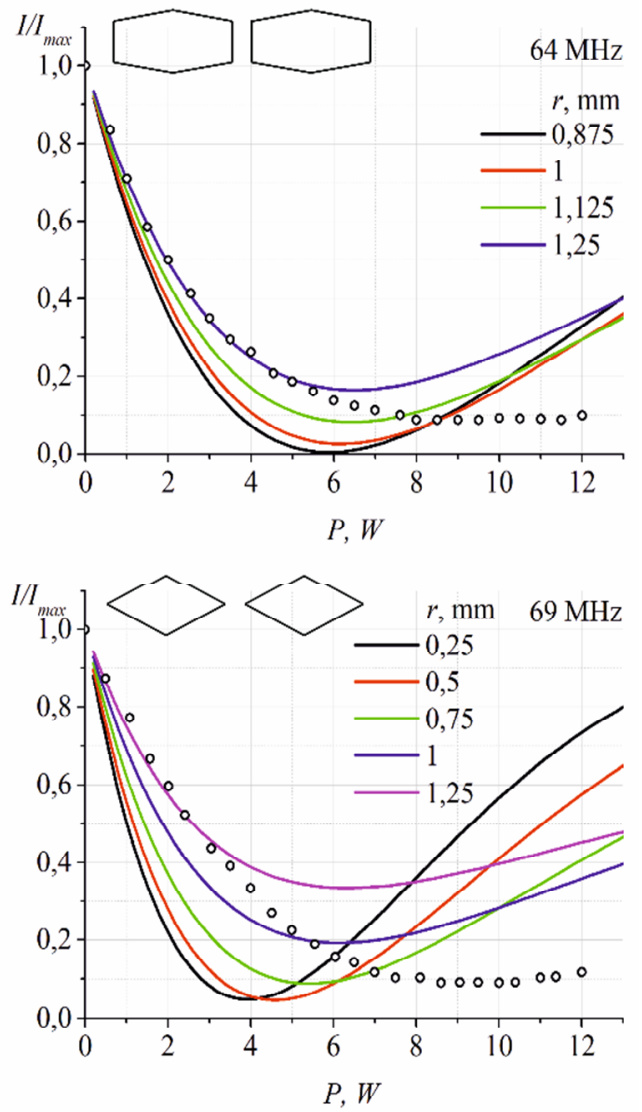

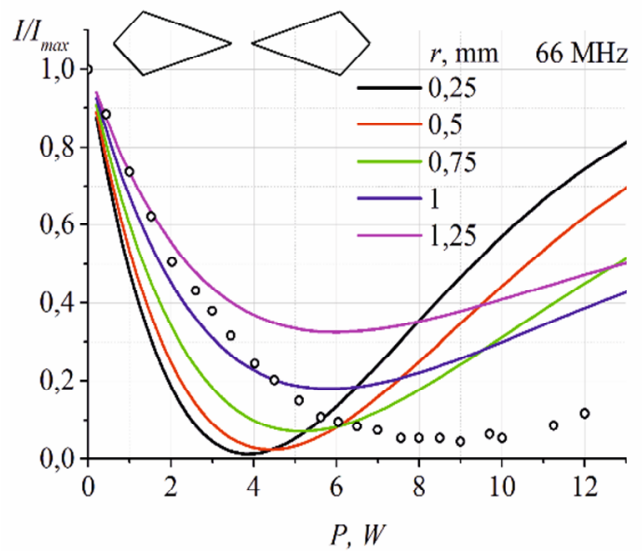

Fig. 11. Dependences of output intensities for a zeroorder light on acoustic power: $r$ denotes a laser-beam radius. Solid lines represent theoretical calculations for different laser-beam radii and dots correspond to experimental results. 
It is seen from Fig. 11 that the maximal diffraction efficiency (i.e., the lowest intensity of the output zero-order beam) increases with decreasing cross-section of light beam. This behaviour occurs due to inhomogeneity-driven reduction of AO-interaction length [10]. The $100 \%$ diffraction efficiency is achievable only in the case of equal interaction lengths for all the partial components in Eq. (16), the same way as in the case of rectangular transducers. The beam-radius reduction leads to decreasing difference between the interaction lengths of the partial components, resulting in higher diffraction efficiency. However, a substantial reduction in the beam radius can impose a negative effect, since a very thin light beam effectively feels any transducer shape as a rectangular one. In such a case the optimal acoustic frequency differs from that defined for a specifically shaped transducer.

There is a single marked disagreement between the theoretical and experimental traces. While the general trends for the traces are similar, the experimental ones seem to suffer from insufficient acoustic power. This might be caused by the losses acquired during electro-acoustic transformation or, alternatively, by some effects of non-uniformity of the acoustic field that appear due to sound diffraction, which has not been taken into account.

\section{Conclusion}

The concept of simultaneous AO diffractions by two acoustic lobes in AO cells with shaped multielement phased-array transducers has been considered. The conditions of such diffraction mode have been defined and a mathematical model for diffraction efficiency, which is based on the assumption of 'sound column', has been developed. In particular, the $\mathrm{AO}$ cells based on $\mathrm{SiO}_{2}$ single crystals with two-element phased-array transducers and a fixed phase shift of $\pi$ have been examined.

Calculations of the acoustic-field distribution in the $\mathrm{SiO}_{2}$ crystals have shown that, in the operational region, the acoustic wavefront remains planar, thus allowing the assumption of 'sound column' to be valid. It is shown that the parallel diffraction regime is almost three times more energy-efficient than the serial regime. Additionally, transducer-shaping technique can be efficiently used to steer the lobes satisfying the condition of simultaneous AO diffractions, when the acoustic frequency and the other parameters of the AO cell are kept fixed. Particularly, the diamond shape has allowed us to adjust the operating frequency within $23 \%$.

\section{Acknowledgments}

This work was performed within the framework of the Grant \#19БФ052-04 from the Ministry of Education and Science of Ukraine.

\section{References}

1. Gordon I E, 1966. A review of acoustooptical deflection and modulation devices. Proc. IEEE. 54: 1391-1400.

2. Pinnow D A, 1971. Acousto-optic light deflection: design considerations for first order beam steering transducers. IEEE Trans. Sonics and Ultrasonics. 18: 209-214.

3. Antonov S, Prokolov V, Rezvov Y and Vainer A, 2014. Two-element phased-array acoustooptic deflector at high diffraction efficiency: scanning range broadening. Universal J. Phys. and Appl. 8: 90-95.

4. Aboujeib J, Perennou A, Quintard V and Bihan J, 2007. Planar phased-array transducers associated with specific electronic command for acousto-optic deflectors. J. Opt. A: Pure \& Appl. Opt. 9: 463-469.

Ukr. J. Phys. Opt. 2021, Volume 22, Issue 3 
5. Peled I, Kaminsky R and Kotler Z, 2015. Acousto-optics bandwidth broadening in a Bragg cell based on arbitrary synthesized signal methods. Appl. Opt. 54: 5065-5073.

6. Pepe D R, Wasilousky P A and Krainak M, 1987. A high performance apodized phased array Bragg cell. Proc. SPIE, Opt. Technol. Microwave Appl. III. 789: 116-126.

7. Bademian L, 1986. Parallel-channel acousto-optic modulation. Opt. Eng. 25: 303-308.

8. Pieper R J and Korpel A, 1983. Matrix formalism for the analysis of acoustooptic beam steering. Appl. Opt. 22: 4073-4081.

9. Balakshy V I, Parygin V N and Chirkov L E. Physical principles of acousto-optics. Moscow: Radio i Sviaz (1985).

10. Voloshinov V B and Knyazev G A, 2003. Acoustooptic cells with nonuniform length of lightsound interaction. Techn. Phys. 48: 1475-1479.

11. Makarov O Yu and Voloshinov V B, 2005. Visualization of back reflected acoustic waves in paratellurite single crystals by means of acousto-optics. Proc. SPIE, Acousto-Optics and Photoacoustics. 5953: 1-10.

12. Yurchenko O, Kolyenov S, Pilgun Yu, Pogorielova G, Polishko O, Smirnov E, 2014. Total internal reflection ultrasonic sensor for detection of subsurface flaws: Research into underlying physics. Final report. http://www.dtic.mil/docs/citations/ADA620159

13. O'Neill B E and Maev R Gr, 1998. Integral approximation method for calculating ultrasonic beam propagation in anisotropic materials. Phys. Rev. B. 58: 5479-5485.

14. Newberry B P and Thompson R B, 1989. A paraxial theory for the propagation of ultrasonic beams in anisotropic solids. J. Acoust. Soc. Amer. 85: 2290-2300.

Illienko M. O., Pilgun Yu. V. and Smirnov Ye. M. 2021. Simultaneous acousto-optic diffractions by two lobes of a shaped phased-array transducer. Ukr.J.Phys.Opt. 22: $138-150$.

doi: $10.3116 / 16091833 / 22 / 3 / 138 / 2021$

Анотація. Розглянуто одночасну дифракиію світла на двох пелюстках діаграми направленості звукового випромінювання в акустооптичному модуляторі, що побудований на монокристалі $\mathrm{SiO}_{2}$ і має двоелементний фазований перетворювач із фіксованим зсувом фаз $\pi$. Впровадження такого типу дифракиії значно поліпшуе енергоефективність пристрою. Зміну форми перетворювача використано для керування пелюстками та задоволення умов Брегга одночасної акустооптичної дифракиії. Визначено умови дифракиії та запропоновано математичну модель для розрахунку ії ефективності. Показано, що паралельний режим дифракції майже втричі енергоефективніший, ніж послідовний режим. Проаналізовано кілька конфігураиій форм елементів перетворювача. Зокрема, показано, що зміна розмірів ромбовидних елементів дає змогу регулювати робочу частоту в межах 23\%. Створено експериментальний зразок пристрою. 